\title{
Stick-Slip Friction of PDMS Surfaces for Bioinspired Adhesives
}

Longjian Xue, ${ }^{*} \uparrow, \ddagger$ Jonathan T. Pham, ${ }^{\ddagger}$ Jagoba Iturri, ${ }^{\ddagger}$ and Aránzazu del Campo ${ }^{\ddagger}, \S$

†School of Power and Mechanical Engineering, Wuhan University, South Donghu Road 8,430072 Wuhan, China

†ax-Planck-Institut für Polymerforschung, Ackermannweg 10, 55128 Mainz, Germany

${ }^{\S}$ INM - Leibniz Institute for New Materials, Campus D2 2. Saarbrücken, Germany

*E-mail: xuelongjian@whu.edu.cn.

Supporting materials includes:

Supporting Video: The video was recorded on pPDMS with a normal loading force of $1 \mathrm{mN}$. The pPDMS moved at a speed of $100 \mu \mathrm{m} / \mathrm{s}$ over $500 \mu \mathrm{m}$ to right and then back.

\section{Supporting Table 1}

\section{Supporting Figure S1, S2, S3}




\section{Supporting Table 1}

Shear stiffness $(\mathrm{mN} / \mu \mathrm{m})$ calculated from the slop of the curve in the static friction part.

\begin{tabular}{ccccc} 
Normal loading & pPDMS & epPDMS & sPDMS & ePDMS \\
\hline $1 \mathrm{mN}$ & 0.2608 & 0.2933 & 0.3190 & 0.3927 \\
$4 \mathrm{mN}$ & 0.3598 & 0.3976 & 0.4509 & 0.4895 \\
$7 \mathrm{mN}$ & 0.4385 & 0.4515 & 0.4920 & 0.5638
\end{tabular}




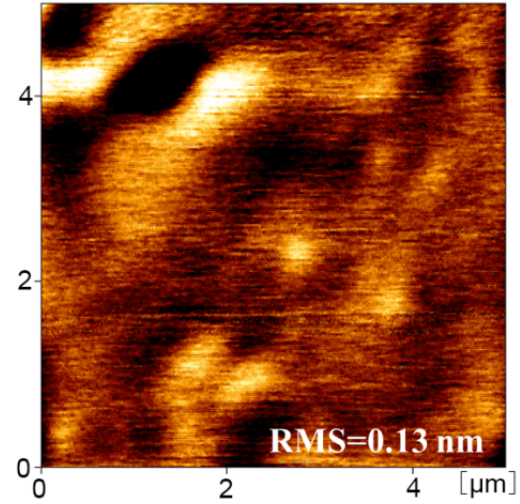

(a)

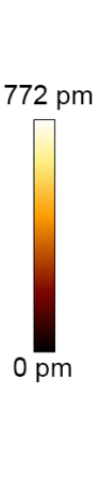

2

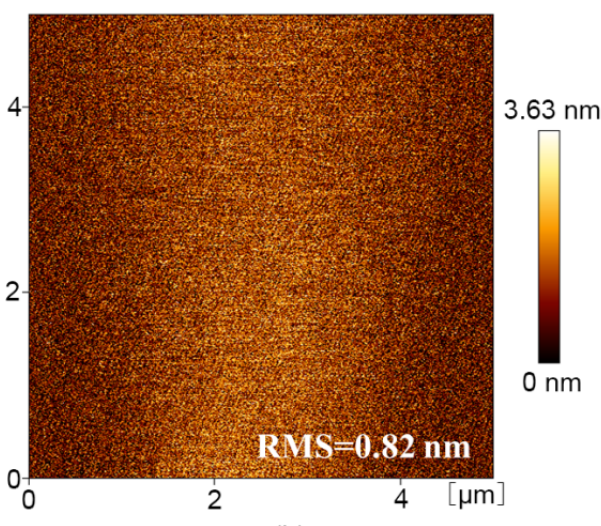

(b)

Figure S1. AFM images of (a) os-PDMS and (b) oe-PDMS. 
(a)

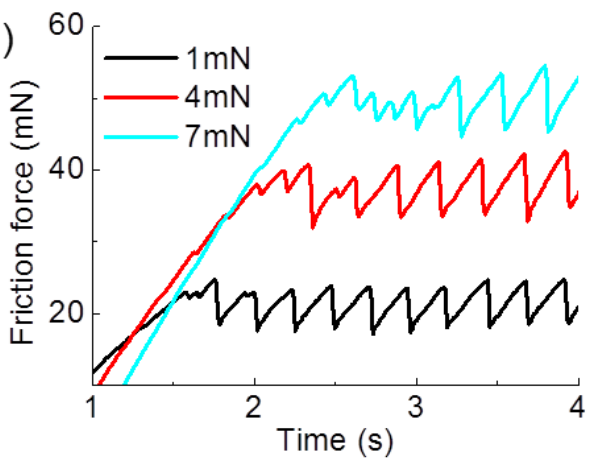

(b)

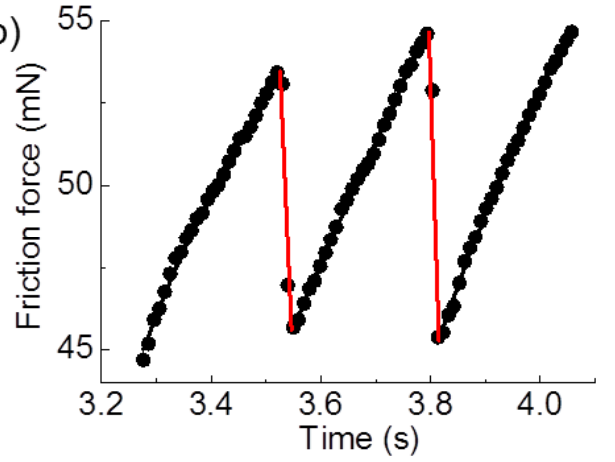

(c)

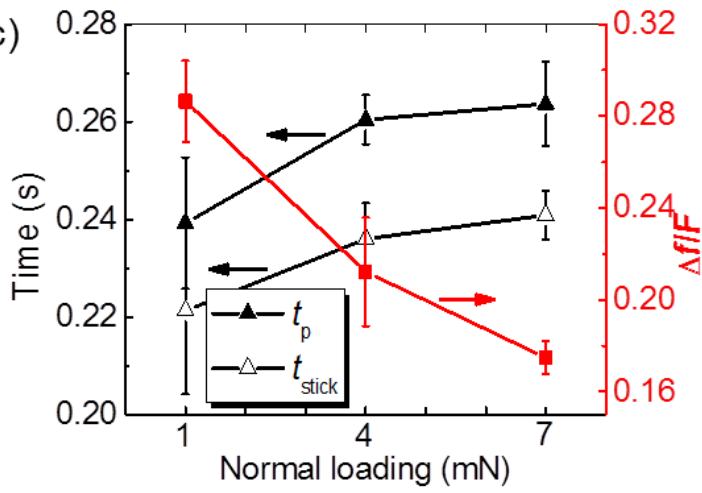

(d)

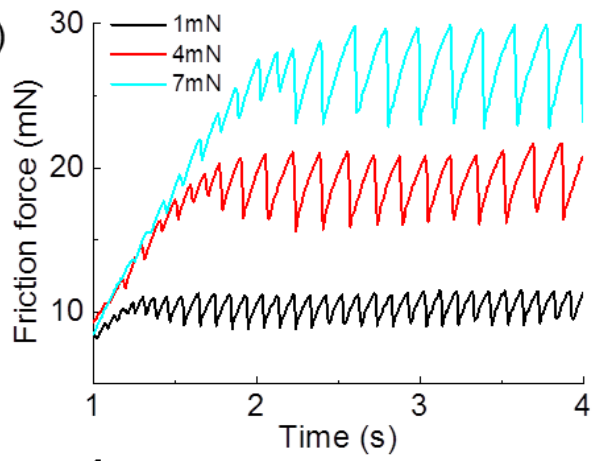

(e)

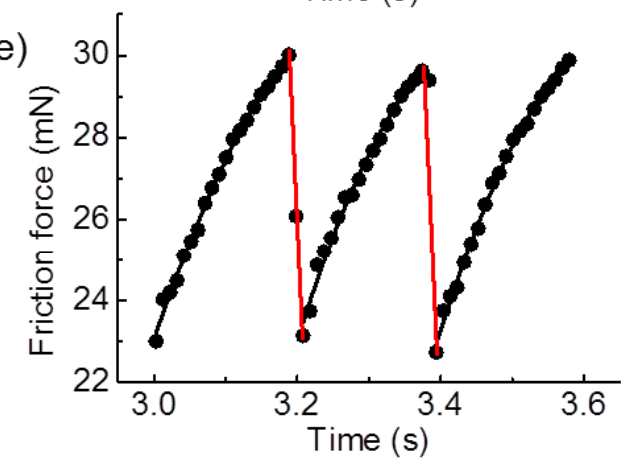

(f)

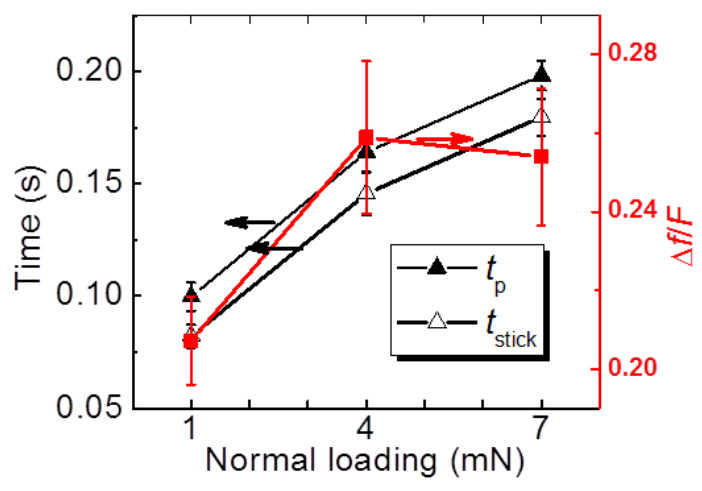

Figure S2. Representative stick-slip motions on (a) oe-PDMS and (d) oep-PDMS at normal loads of 1, 4 and $7 \mathrm{mN}$. Detailed stick-slip patterns (pattern III) on (b) oe-PDMS and (e) oepPDMS. (c, f) Dependence of $t_{\text {stick, }}, t_{\mathrm{p}}$ and $\Delta f f F$ of (c) oe-PDMS and (f) oep-PDMS on normal loading force. The gap between $t_{\text {stick }}$ and $t_{\mathrm{p}}$ defines the value of $t_{\text {slip. }}$. Each data point in panel (c) and (f) represents measurements of 10 stick-slip periods. The error bars indicate standard deviations. 


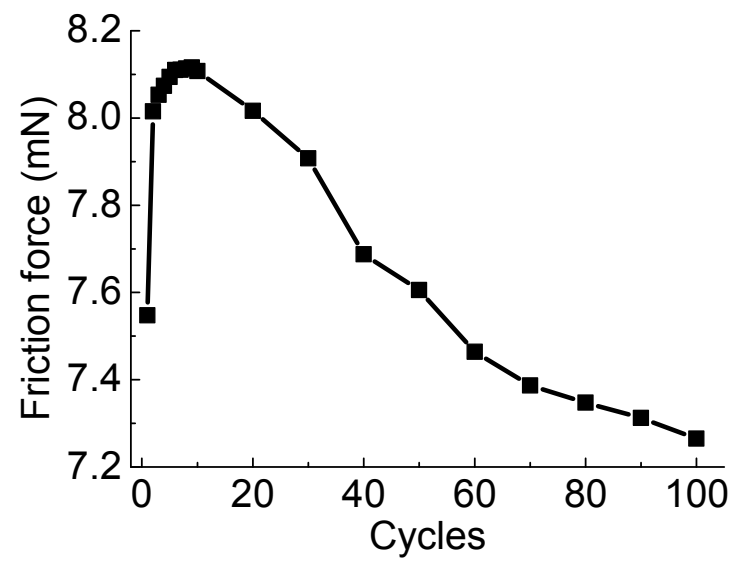

Figure S3. The dependence of friction force on the repeating cycles on s-PDMS. When a clean probe is brought into contact with s-PDMS, the oligomers will be transferred to the surface of the probe. The linking of oligomers between s-PDMS and the probe can enhance the friction force. The saturation of oligomers on the probe surface and the alignment of oligomers at the contact interface during repeating shearing reduce the friction force as the movable oligomers serve as the lubricant. 